\title{
Anotaciones breves sobre el síndrome de liberación de citocinas y el bloqueo terapéutico de la interleucina-6 en SARS-CoV-2/COVID-19
}

\author{
Brief annotations on cytokine release syndrome and interleukin-6 \\ therapeutic blockage in SARS-CoV-2/COVID-19 \\ Luis M Amezcua-Guerra*
}

\section{INTRODUCCIÓN}

$\mathrm{H}$ asta el 22 de abril de 2020, la enfermedad por coronavirus 2019 (COVID-19), causada por el coronavirus 2 del síndrome respiratorio agudo grave (SARS-CoV-2), ha sido confirmada en 2'637,717 individuos en todo el mundo, y ha ocasionado una mortalidad aproximada de 184,225 pacientes. En este momento hay 1'735,702 con infección activa, $56,680(\sim 3 \%)$ con una enfermedad grave o crítica. ${ }^{1}$ La principal causa de muerte en ellos es el síndrome de distrés respiratorio agudo (ARDS), habitualmente en conjunto con daño miocárdico y niveles séricos inusitadamente elevados de mediadores inflamatorios. ${ }^{2} \mathrm{La}$ presencia de concentraciones elevadas de mediadores inflamatorios en circulación es un fenómeno frecuentemente denominado síndrome de liberación de citocinas o tormenta de citocinas. ${ }^{3}$

\section{SÍNDROME DE ACTIVACIÓN DE MACRÓFAGOS}

La linfohistiocitosis hemofagocítica es una condición aberrante de respuesta inmunitaria hiperinflamatoria e hiperferritininémica, dirigida por diferentes subpoblaciones de linfocitos T y asociada al síndrome de liberación de citocinas. El término síndrome de activación de macrófagos (MAS) se refiere a un subgrupo de pacientes con linfohistiocitosis hemofagocítica secundaria, en un contexto de autoinflamación o autoinmunidad sistémica. ${ }^{4}$ En 2004 se realizó una actualización de los criterios diagnósticos de linfohistiocitosis hemofagocítica (Tabla 1). Estos criterios consideran la presencia de un diagnóstico molecular consistente con linfohistiocitosis como dato suficiente para su diagnóstico; en este sentido, es menester recordar que la linfohistiocitosis primaria comprende a un grupo de alteraciones de la inmunorregulación por defectos genéticos, e incluyen padecimientos como el síndrome de Griscelli o el síndrome de Chediak-Higashi. Para ser considerado el diagnóstico de MAS, un individuo debe cumplir al menos cinco de ocho criterios clínicos y de laboratorio. En condiciones excepcionales, se puede considerar la existencia de MAS e iniciar terapias dirigidas aun sin cumplirse todos los criterios. ${ }^{4}$

EI MAS es una condición hiperinflamatoria asociada a diferentes disparadores, incluye infecciones, enfermedades autoinmunes y neoplasias. Característicamente, se ha observado la inducción de MAS en infecciones por el virus de Epstein-Barr, influenza H1N1 o citomegalovirus, así como por los coronavirus causantes del SARS (Severe Acute Respiratory Syndrome) y el MERS (Middle East Respiratory Syndrome). El MAS se caracteriza por fiebre, hepatoesplenomegalia, citopenias, niveles elevados de ferritina, triglicéridos, deshidrogenasa láctica, dímero D y aminotransferasas, así como hipofibrinogenemia. La hemofagocitosis típicamente está ausente al inicio de la enfermedad, aunque usualmente ocurre en algún punto de la evolución. La fase aguda del MAS refleja un estado de activación inmunitaria sistémica, con niveles extraordinariamente elevados de 
citocinas como interleucina-6 (IL-6), IL-1 $\beta$, IL-2, IL-12, IL-18, factor de necrosis tumoral (TNF) e interferón gamma (IFN- $\gamma) \cdot{ }^{3}$ Esta tormenta de citocinas desencadena una cascada de vías inflamatorias que, si no es limitada, lleva a grave daño tisular y muerte. ${ }^{5}$

Se ha desarrollado un sistema de puntuación clínico y de laboratorio para calcular la probabilidad de padecer linfohistiocitosis hemofagocítica reactiva, que puede ser una herramienta útil en el diagnóstico de MAS. ${ }^{6}$ Éste incluye el antecedente de inmunosupresión/ inmunodeficiencia subyacente, temperatura corporal, hepatomegalia, esplenomegalia, conteo leucocitario y plaquetario, niveles de hemoglobina y ferritina, triglicéridos, fibrinógeno, aminotransferasas y la presencia de hemofagocitosis en el aspirado de médula ósea. Una versión electrónica de esta calculadora se encuentra disponible en línea de manera gratuita (http://saintantoine.aphp.fr/score/).

\section{LA INTERLEUCINA-6 EN EL SÍNDROME DE LIBERACIÓN DE CITOCINAS}

La infección por parte de los coronavirus a los monocitos, macrófagos y células dendríticas da como resultado su activación y posterior

Tabla 1: Criterios para el diagnóstico de linfohistiocitosis hemofagocítica (LHH).

A. Un diagnóstico molecular consistente con LHH

B. Cinco de los ocho listados abajo

1) Fiebre

2) Esplenomegalia

3) Citopenias (afectando al menos dos linajes)

a) Hemoglobina $<90 \mathrm{~g} / \mathrm{L}$

b) Plaquetas $<100,000 \times 10^{9} / \mathrm{L}$

c) Neutrófilos $<1.0 \times 10^{9} / \mathrm{L}$

4) Hipertrigliceridemia $y / o$ hipofibrinogenemia

a) Triglicéridos $\geq 265 \mathrm{mg} / \mathrm{dL}$

b) Fibrinógeno $\leq 1.5 \mathrm{~g} / \mathrm{L}$

5) Hemofagocitosis en el aspirado de médula ósea 0 en bazo 0 en linfonodos. En ausencia de malignidad

6) Actividad baja o nula de células asesinas naturales

7) Ferritina $\geq 500 \mu \mathrm{g} / \mathrm{L}$

8) CD25 soluble (receptor de IL-2) $\geq 2,400 \mathrm{U} / \mathrm{mL}$ secreción de IL-6 y otras citocinas. ${ }^{7}$ En particular, la IL-6 es una citocina prototípica de este proceso inflamatorio, que señala a través de dos vías principales denominadas señalización cis y trans. En el sistema de señalización cis, la IL-6 se une al receptor de IL-6 unido a la membrana celular, en un complejo multimolecular con la glucoproteína gp130. Una vez conformado el complejo, se inicia la transducción de señales corriente abajo, mediada por las cinasas Janus (JAK) y el transductor de señales (STAT3). De manera interesante, la gp130 se expresa en múltiples estirpes celulares, mientras que la expresión del receptor de IL-6 unido a membrana está restringida básicamente a células de estirpe inmunitaria. La activación de la señalización cis produce efectos pleiotrópicos en células del sistema inmunitario adaptativo (linfocitos $\mathrm{T}$ y B) e innato (neutrófilos, macrófagos y células asesinas naturales), por lo que puede contribuir en el síndrome de liberación de citocinas. $^{8}$

En el sistema de señalización trans, las concentraciones elevadas de IL-6 en circulación permiten que ésta se una a la forma soluble del receptor de IL-6, formando un complejo directo con dímeros de glucoproteína gp130 sobre la superficie de, prácticamente, cualquier célula. Así, la cascada de señalización resultante se puede activar en células que no expresan receptor de IL-6 unido a la membrana, tal como las células endoteliales. Esto da como resultado una verdadera tormenta de citocinas, que implica la secreción sistémica de moléculas inflamatorias, quimioatrayentes y vasoactivas como el factor de crecimiento del endotelio vascular (VEGF), las quimiocinas CCL2 (MCP-1, monocyte chemoattractant protein-1) e IL-8, y cantidades adicionales de IL-6. En conjunto, esta liberación masiva de citocinas facilita la permeabilidad vascular y la extravasación de líquido, participando de manera activa en la fisiopatología de la hipotensión arterial y la disfunción pulmonar en el ARDS. ${ }^{9}$

La eficacia de los antagonistas de IL-6 en el tratamiento del MAS, específicamente aquéllos dirigidos contra el receptor de IL-6, enfatiza el papel central de la señalización de IL-6 en la fisiopatología de los síndromes hiperinflamatorios inducidos por citocinas. ${ }^{7}$ 


\section{SÍNDROME DE LIBERACIÓN DE CITOCINAS EN PACIENTES CON COVID-19}

En un estudio que incluyó a 41 pacientes con infección confirmada por SARS-CoV-2 provenientes de Wuhan, China, se encontraron concentraciones plasmáticas elevadas de IL-1 $\beta$, antagonista del receptor de IL-1 (IL-1RA), IL-7, IL-8, IL-9, IFN- $\gamma$, TNF, factores de crecimiento fibroblástico (FGF), estimulante de colonias de granulocitos (G-CSF), estimulante de colonias de granulocitos y macrófagos (GM-CSF), de crecimiento derivado de plaquetas (PDGF) y VEGF, quimiocinas CXCL10 (IP-10, interferon gamma-induced protein 10), CCL2, CCL3 (MIP1 - $\alpha$, macrophage inflammatory protein 1-alpha) y CCL4 (MIP-1- $\beta$, macrophage inflammatory protein 1-beta) en comparación con individuos sanos. Trece pacientes (32\%) requirieron ingreso a la unidad de cuidados intensivos (UCl), quienes mostraron niveles plasmáticos más elevados de IL-2, IL-7, IL-10, G-CSF, IP-10, MCP-1, MIP-1- $\alpha$ y TNF en comparación con aquéllos que no requirieron manejo en $\mathrm{UCl}{ }^{10}$

En un análisis sobre predictores de muerte en 150 pacientes con COVID-19 realizado en Wuhan, China, se identificaron niveles elevados de proteína C reactiva $(126.6 \pm 106.3 \mathrm{mg} / \mathrm{L}$ vs $34.1 \pm 54.5 \mathrm{mg} / \mathrm{L} ; \mathrm{p}<0.001), \mathrm{IL}-6(11.4 \pm 8.5$ $\mathrm{ng} / \mathrm{mL}$ vs $6.8 \pm 3.6 \mathrm{ng} / \mathrm{mL} ; \mathrm{p}<0.001)$ y ferritina $(1,297 \pm 1,030 \mathrm{ng} / \mathrm{mL}$ vs $614 \pm 752 \mathrm{ng} / \mathrm{mL}$; $p<0.001$ ) en los pacientes que fallecieron. ${ }^{2}$

En una caracterización clínica e inmunológica de 21 pacientes con COVID-19 provenientes de Wuhan, se identificó que los casos graves tenían linfopenia con mayor frecuencia, hipoalbuminemia, hipertransaminasemia, así como niveles elevados de proteína $C$ reactiva, ferritina, dímero D, receptor soluble de IL-2, IL-6, IL-10 y TNF, en comparación con los pacientes con enfermedad moderada. Además, se encontraron conteos disminuidos de linfocitos $\mathrm{T}$, células CD4 + y CD8+ en prácticamente todos, aunque estos conteos fueron particularmente bajos en los pacientes con enfermedad más grave. ${ }^{11}$

\section{PERSPECTIVAS A CORTO PLAZO}

En aras de reducir la mortalidad de pacientes con COVID-19, se necesitan esfuerzos estratégicos que promuevan el uso de terapias que limiten el síndrome de liberación de citocinas asociado a la infección por SARS-CoV-2. El uso de fármacos dirigidos contra el eje de IL-6, como el tocilizumab, el sarilumab y el siltuximab para manejar el síndrome de liberación de citocinas es un nuevo y excitante campo. De hecho, los resultados preliminares de un estudio abierto realizado en 21 pacientes con COVID-19 en estado grave o crítico, tratados con tocilizumab (anticuerpo monoclonal dirigido contra el receptor soluble de IL-6) son alentadores. ${ }^{12}$ La fiebre disminuyó en todos los pacientes durante el primer día que recibieron tocilizumab. Los requerimientos de oxígeno se redujeron en $75 \%$ de ellos y se observó mejoría en las lesiones pulmonares evaluadas por tomografía en $90 \%$ de los pacientes. Más aún, 90\% fueron egresados del hospital a los 13.5 días de iniciado el tocilizumab. Muchas otras opciones terapéuticas encaminadas a modificar la liberación de citocinas se están trasladando a ensayos clínicos para COVID-19. Además del obvio uso de glucocorticoides, estrategias diversas como el uso de los antimaláricos (hidroxicloroquina, cloroquina), antibióticos (azitromicina) y antiparasitarios (ivermectina), el bloqueo selectivo de citocinas como TNF (adalimumab, etanercept) o IL-1 $\beta$ (canakinumab, anakinra), la citoablación (rituximab), el uso de inmunoglobulina intravenosa, la adsorción de citocinas, el plasma de pacientes convalecientes y la inhibición de los sistemas JAK/STAT están abriendo nuevas perspectivas terapéuticas con potencial uso clínico en esta pandemia.

Finalmente, es recomendable que todos los pacientes con COVID-19 sean sometidos a pruebas de detección de hiperinflamación, incluyendo marcadores de fase aguda asequibles en laboratorios generales, tal como los niveles séricos o plasmáticos de ferritina, proteína $C$ reactiva, fibrinógeno y dímero $D$, así como la relación del conteo de linfocitos/plaquetas y la velocidad de sedimentación globular. ${ }^{13}$ Además, el uso sistemático de herramientas clinimétricas para evaluar la probabilidad de desarrollo del síndrome de linfohistiocitosis hemofagocítica (p. ej., la calculadora HScore) puede facilitar la identificación temprana de subgrupos de pacientes con COVID-19, en quienes la inmunosupresión temprana podría mejorar los desenlaces clínicamente relevantes y la supervivencia. ${ }^{6}$ 


\section{REFERENCIAS}

1. Worldometer. Coronavirus cases. Worldometer. doi: 10.1101/2020.01.23.20018549V2.

2. Ruan Q, Yang K, Wang W, Jiang L, Song J. Clinical predictors of mortality due to COVID-19 based on an analysis of data of 150 patients from Wuhan, China. Intensive Care Med. 2020 Mar 3. doi: 10.1007/ s00134- 020-05991-x. [Epub ahead of print]

3. Rosário C, Zandman-Goddard G, Meyron-Holtz EG, D'Cruz DP, Shoenfeld Y. The hyperferritinemic syndrome: macrophage activation syndrome, Still's disease, septic shock and catastrophic antiphospholipid syndrome. BMC Medicine. 2013; 11 (1): 185.

4. La Rosée P, Horne AC, Hines M, von Bahr Greenwood T, Machowicz R, Berliner N et al. Recommendations for the management of hemophagocytic lymphohistiocytosis in adults. Blood. 2019; 133 (23): 2465-2477.

5. Strippoli R, Caiello I, De Benedetti F. Reaching the threshold: a multilayer pathogenesis of macrophage activation syndrome. J Rheumatol. 2013; 40 (6): 761-767.

6. Fardet L, Galicier L, Lambotte O, Marzac C, Aumont C, Chahwan D et al. Development and validation of the HScore, a score for the diagnosis of reactive hemophagocytic syndrome. Arthritis Rheumatol. 2014; 66 (9): 2613-2620.

7. Moore JB, June $\mathrm{CH}$. Cytokine release syndrome in severe COVID-19. Science. 2020; 368 (6490): 473-474.

8. Kang S, Tanaka T, Narazaki M, Kishimoto T. Targeting interleukin-6 signaling in clinic. Immunity. 2019; 50 (4): 1007-1023.

9. Tanaka T, Narazaki M, Kishimoto T. Immunotherapeutic implications of IL-6 blockade for cytokine storm. Immunotherapy. 2016; 8 (8): 959-970.

10. Huang C, Wang Y, Li X, Ren L, Zhao J, Hu Y et al. Clinical features of patients infected with 2019 novel coronavirus in Wuhan, China. Lancet. 2020; 395 (10223): 497-506.

11. Chen G, Wu D, Guo W, Cao Y, Huang D, Wang H et al. Clinical and immunologic features in severe and moderate coronavirus disease 2019. J Clin Invest. 2020; 130 (5): 2620-2629.

12. Effective Treatment of Severe COVID-19 Patients with tocilizumab [Internet]. ChinaXiv [fecha de publicación: 3 de mayo de 2020]. Disponible en: http://www. chinaxiv.org/abs/202003.00026

13. Mehta P, McAuley DF, Brown M, Sanchez E, Tattersall RS, Manson JJ; HLH Across Speciality Collaboration, UK. COVID-19: consider cytokine storm syndromes and immunosuppression. Lancet. 2020; 395 (10229): 1033-1034.

\section{Correspondencia:}

Luis M Amezcua-Guerra

Departamento de Inmunología,

Instituto Nacional de Cardiología

«lgnacio Chávez».

Juan Badiano Núm. 1,

Col. Belisario Domínguez, Sección XVI,

Tlalpan 14080, Ciudad de México, México.

E-mail: Imamezcuag@gmail.com

\section{RESPONSABILIDADES ÉTICAS}

Protección de personas y animales. Los autores declaran que para esta investigación no se han realizado experimentos en seres humanos ni en animales.

Confidencialidad de los datos. Los autores declaran que han seguido los protocolos de su centro de trabajo sobre la publicación de datos de pacientes.

Derecho a la privacidad y consentimiento informado. Los autores han obtenido el consentimiento informado de los pacientes $y / o$ sujetos referidos en el artículo. Este documento obra en poder del autor de correspondencia. 